\title{
Decreased serum IL-27 and IL-35 levels are associated with disease severity in neuromyelitis optica spectrum disorders
}

Da-Qi Zhang ${ }^{\mathrm{a}}$, Kun Jia ${ }^{\mathrm{a}}$, Rong Wang ${ }^{\mathrm{b}}$, Ting Li ${ }^{\mathrm{a}}$, Ning Zhao ${ }^{\mathrm{a}}$, Li-Na Yang ${ }^{\mathrm{a}}$, Li Yang ${ }^{\mathrm{a}}$

${ }^{a}$ Department of Neurology and Tianjin Neurological Institute, Tianjin Medical

University General Hospital, Tianjin 300052, China

${ }^{\mathrm{b} S} \mathrm{School}$ of Integrative Medicine, Tianjin University of Traditional Chinese Medicine, Tianjin 300193, China

Corresponding author: Li Yang, M.D., Ph.D.

Department of Neurology and Tianjin Neurological Institute Tianjin Medical University General Hospital

Tianjin 300052, China

Tel.: +86-22-608-14536. Fax: +86-22-608-17471

Email: yangli2001@tmu.edu.cn \& yungli2001@yahoo.com

$1 / 19$ 


\begin{abstract}
The interleukin 12 (IL-12) family plays important roles in autoimmune diseases. To explore the roles of the IL-12 family members IL-27 and IL-35 in the pathogenesis of neuromyelitis optica spectrum disorders (NMOSD), we determined their serum and cerebral spinal fluid levels and assessed potential correlations with clinical characteristics. Serum IL-27 levels were negatively correlated with disease severity and spinal cord lesion length, while serum IL-35 levels were negatively correlated with disease severity and annual relapse rate. Thus, IL-27 and IL-35 may be important biomarkers of NMOSD severity and these molecules might represent potential therapeutic cytokines for treating NMOSD.
\end{abstract}

Keywords: IL-12; IL-27; IL-35; neuromyelitis optica spectrum disorders (NMOSD) 


\section{Introduction}

Neuromyelitis optica spectrum disorders (NMOSD) have been defined as rare, disabling autoimmune spectrum diseases of the central nervous system, which are distinct from multiple sclerosis (MS). NMOSD comprise neuromyelitis optica (NMO), longitudinally extensive myelitis (LETM), optic neuritis (ON), and other typical demyelinating brain syndromes involving the area postrema, periependymal brainstem, diencephalon, and cerebrum(Wingerchuk et al., 2015). Great progress in our understanding of the pathogenesis of NMOSD has been made since the discovery that aberrant anti-aquaporin 4 antibodies (AQP4 Ab) in the serum of patients with NMOSD underlie many disease phenotypes(Jarius and Wildemann, 2010; Lennon et al., 2004).

In addition to AQP4 Ab, recent studies have demonstrated that various cytokines and chemokines may be involved in immune responses and pathogenesis of peripheral/central nervous system in patients with NMO such as IL-6, IL-17, IL-10, IL-12, IFN- $\gamma$, BAFF, CXCL13 and TNF- $\alpha$ (Matsushita et al., 2013; Uzawa et al., 2010; Uzawa et al., 2014; Zhong et al., 2011). However, the role of newly recognized members of the interleukin12 (IL-12) family, such as IL-27 and IL-35, remains unclear. It is known that members of the IL-12 family, including IL-12, IL-23, IL-27 and IL-35, play important roles in regulating immune responses and the pathology of inflammatory and autoimmune diseases(Sun et al., 2015; Vignali and Kuchroo, 2012).

Recent studies have demonstrated that IL-12 and IL-23 are pro-inflammatory cytokines that are mainly secreted by dendritic cells (DCs) and macrophages. In turn, these molecules can induce the production of a number of cytokines, regulate a variety of effector cells, and contribute to the pathogenesis of CNS autoimmune diseases(Becher et al., 2003; Comabella et al., 1998; Cua et al., 2003; Vignali and Kuchroo, 2012). IL-27 is a newly recognized member of the IL-12 family composed of Epstein-Barr virus-induced gene protein 3 (EBI3) and p28 subunits, which is often produced by activated antigen presenting cells. Intriguingly, IL-27 can play both pro-inflammatory and anti-inflammatory roles(Batten et al., 2006; Cox et al., 2011; Fitzgerald et al., 2007). IL-35 is similar to IL-27 in that it is a heterodimeric cytokine 
encompassing the p35 subunit of IL-12 and EBI3. IL-35 is secreted by activated inflammatory cells, such as anti-CD3/CD28-induced Treg cells, activated dendritic cells, and macrophages. IL-35 has been shown to perform an immunomodulatory role in a wide variety of disease conditions(Collison et al., 2010; Collison et al., 2007; Wang et al., 2014).

However, possible roles of IL-27 and IL-35 in NMOSD are unknown. Therefore, in the present study, we determined the levels of IL-27 and IL-35 in the serum and cerebrospinal fluid (CSF) of patients with NMOSD and explored if there were relationships between these two newly recognized IL-12 members and clinical parameters.

\section{Methods}

\subsection{Subjects}

Forty-five patients with NMOSD were recruited from the Neurology Department of Tianjin Medical University General Hospital from January 2012 to July 2015. The patients were diagnosed according to the criteria defined by Wingerchuk et al.(Wingerchuk, et al., 2015). All patients with NMOSD were treated with intravenous methylprednisolone at a dose of $0.5 \mathrm{~g} /$ day for $3-5$ consecutive days during acute exacerbations, and then a subset were treated with low-dose prednisolone and/ or azathioprine at a dose of $0.1 \mathrm{~g} /$ day during the initial two years of remission phases. As a serum control group, we enrolled forty healthy age- and sex-matched individuals from the Health Care Center of our hospital. In addition, we enrolled 19 patients with other non-inflammatory neurological disorders (ONNDs) as CSF controls, comprising 6 patients with subacute combined degeneration of the spinal cord, 11 patients with motor neuron disease, and 2 patients with Wernicke-Korsakoff syndrome.

All the research procedures were approved by the ethics committee of Tianjin Medical University General Hospital, and all the participants provided written informed consent prior to participation.

\subsection{Clinical assessment}


The data for demographic features, clinical symptoms, Kurtzke Expanded Disability Status Scale (EDSS) score at sampling, annual relapse rate during the disease course (number of relapses per year), and CSF findings including cells, protein and oligoclonal bands, were acquired from our internal database system. The EDSS was evaluated independently by two neurologists who were certified for competency in EDSS scoring by Neurostatus (Neurostatus Systems AG, Basel, Switzerland). Conventional and/or enhanced MRI of brain and spinal cord were all performed within 7 days of attack to identify new lesions.

A total of 45 serum samples and $21 \mathrm{CSF}$ samples matched with serum samples were obtained during acute exacerbations. Twelve serum and 9 CSF samples were collected at the patient's first NMOSD attack. All the serum samples were collected before high-dose intravenous methylprednisolone for acute exacerbations; 26 of these samples were collected from patients who had not received low-dose oral prednisolone and/or azathioprine therapy within at least three months before starting with sampling. Only 10 CSF samples were collected before the administration of any immunosuppressive therapy. All samples were stored at $-80^{\circ} \mathrm{C}$ until analysis.

\subsection{Detection of anti-AQP4 antibodies}

Serum AQP4 antibody was detected by both CBA and fluorescence immunoprecipitation assay (FIPA)(Wang et al., 2015; Yang et al., 2014). The EGFP tagged human AQP4 plasmid and the EGFP plasmid were donated by Professor Angela Vincent and Professor David Beeson (Nuffield Department of Clinical Neurosciences, University of Oxford).

\subsection{Measurement of IL-27and IL-35 levels}

Serum and CSF IL-27 levels were measured using a human IL-27 enzyme-linked immunosorbent assay (ELISA) kit (eBioscience, San Diego, CA, USA) according to the manufacturer's instructions. We also detected serum and CSF IL-35 levels with a human IL-35 enzyme-linked immunosorbent assay (ELISA) kit (Biolegend, San Diego, CA,USA) according to the manufacturer's instructions. The absorbance values of the corresponding substrate were read at $450 \mathrm{~nm}$. IL-27 and IL-35 levels were calculated by referencing to a standard curve, and the lowest detectable levels were 
$9.5 \mathrm{pg} / \mathrm{mL}$ and $0.13 \mathrm{ng} / \mathrm{mL}$, respectively. If the serum or CSF levels were below the lowest detectable levels, we recorded the concentration of serum or CSF IL-27 or IL-35 as zero.

\subsection{Statistical analyses}

Unpaired t-tests or Mann-Whitney U tests were used forcomparisons of continuous variables. Spearman's rank correlation analysis was used for analysis of the associations between IL-27 or IL-35 and clinical parameters. All statistical analyses were performed using GraphPad PRISM 5.0 software (Graph Pad Software Inc., San Diego, CA, USA). P $<0.05$ was considered to be statistically significant in two-tailed tests.

\section{Results}

\subsection{Demographic and clinical features of subjects with NMOSD}

The presenting demographic and clinical features of the 45 patients with NMOSD included in the study are shown in Table 1. All subjects belonged to the Chinese Han ethnic group.

\subsection{Serum and CSF IL-27 and IL-35 levels in NMOSD}

We determined IL-27 and IL-35 levels in patients with NMOSD and healthy controls to explore the role of IL-27- and IL-35-mediated inflammation in the pathogenesis of NMOSD. Our results indicated that serum IL-27 levels were significantly decreased in patients with NMOSD compared to controls $(\mathrm{p}<0.001$, Fig. 1). Given that subset of the enrolled patients had received low-dose oral immunosuppressive treatments before sampling, we compared IL-27 levels in the medicated versus unmedicated groups prior to sampling. We found that IL-27 levels in the medicated group were higher than in the unmedicated group, but the difference did not meet the level of statistical significance $(\mathrm{p}=0.168)$. We further analyzed serum IL-27 levels in anti-AQP4 antibody-positive patients and anti-AQP4 antibody-negative patients, we found that serum IL-27 levels were similar between 
these two groups ( $\mathrm{p}=0.338)$. IL-27 levels in both the anti-AQP4 antibody-positive and anti-AQP4 antibody-negative groups were lower than in control subjects ( $\mathrm{p}=0.004$, $\mathrm{p}=0.005$, respectively, Fig. 1). Similarly, we found that serum IL-35 levels in patients with NMOSD were significantly lower than in controls (p=0.009, Fig. 2), and there was no statistical difference of IL-35 levels between the medicated and unmedicated groups before sampling ( $\mathrm{p}=0.079)$. Serum IL-35 levels in anti-AQP4 antibody-positive patients were significantly decreased compared to control subjects ( $\mathrm{p}=0.017$, Fig. 2). Although serum IL-35 levels in anti-AQP4 antibody-negative patients were also lower than in control subjects, the difference was not statistically significant $(\mathrm{p}=0.096)$. There was likewise not a significant difference in serum IL-35 levels between patients with anti-AQP4 antibody-positive and anti-AQP4 antibody-negative findings ( $\mathrm{p}=0.942)$. CSF IL-27 and IL-35 levels were both below the minimum detectable level in patients with NMOSD and in control subjects.

\subsection{Correlation between serum IL-27 or IL-35 levels and disease severity}

To explore potential associations between IL-27 and IL-35 with disease severity, we determined if there were correlations between serum IL-27 or IL-35 levels and clinical and MRI indications of disease status. We found that serum IL-27 levels were negatively correlated with EDSS and total length of newly identified spinal cord lesion at sampling in NMOSD $(r=-0.314, p=0.035$, Fig. $3 \mathrm{~A} ; \mathrm{r}=-0.387, \mathrm{p}=0.009$, Fig. 3C, respectively). However, no significant correlations between IL-27 levels and annual relapse rate or AQP4 antibody levels (FU) determined by FIPA were found in patients with NMOSD ( $r=-0.173, p=0.255$, Fig. 3D; $r=-0.056, p=0.715$, respectively). We also found a significant negative correlation of IL-35 levels with EDSS and annual relapse rate in NMOSD (r=-0.332, p=0.026, Fig. 4A; r =-0.331, p =0.027, Fig. 4C, respectively). However, there were no significant correlations between serum IL-35 and total length of newly identified spinal cord lesion at sampling or AQP-4 antibody levels (FU) as determined by FIPA ( $r=0.035$, p=0.818, Fig. 4D; r =-0.069, p $=0.653$, respectively). Considering that EDSS obtained in the relapse phase does not necessarily represent the severity of disease due to severe residual symptoms, we 
further analyzed possible associations between IL-27 or IL-35 and EDSS in the 12 patients with first attack of NMOSD. We found a negative correlation between IL-27 or IL35 levels and EDSS of greater statistical significance $(r=-0.600, p=0.039$, Fig. 3B; $r=-0.702, p=0.011$, Fig. 4B, respectively).

\section{Discussion}

It has been reported that cytokine interactions are involved in pathophysiological processes of NMOSD. Recent studies have shown IL-12 family cytokines play important roles in inflammatory processes and are potential therapeutic targets in CNS autoimmune diseases(Sun et al., 2015). In our study, we found that serum IL-27 and IL-35 levels were decreased in patients with NMOSD, indicating that they may be involved in NMOSD pathophysiology.

Recent studies have reported that IL-27 acts as both a pro-inflammatory and inhibitory cytokine. IL-27 has been shown to play a significant role in autoimmune diseases including systemic lupus erythematosus, rheumatoid arthritis, and MS(Li et al., 2010; Tang et al., 2015; Wong et al., 2010), mostly operating as an inhibitor of inflammation. Our results similarly suggest that IL-27 may play a protective role in NMOSD. Despite an increasing number of studies concerning IL-27 pathophysiology, the reasons and mechanisms of decreased IL-27 in the process of NMOSD inflammation remains poorly understood. Previous works suggest that IL-27 might act as a neuroprotectant in NMOSD via its roles in T-cell regulation. It has been demonstrated that IL-27 suppresses inflammation mainly by promoting Th1 differentiation from naïve T-cells. This thereby inhibits the differentiation and function of Th17 cells, which produce pro-inflammatory cytokines, such as IL-17 and IL-21, and leads to an expansion of inducible regulatory T cells that produce IL-10(Batten, Li, 2006; Stumhofer et al., 2006). Moreover, IL-17 and IL-21 increase the severity of inflammation in patients with NMOSD(Wang et al., 2011). In keeping with a role of IL-27 regulation of T-cells in demyelinating immune disorders, recent studies have shown that there is an inverse correlation between plasma and mRNA expression levels of IL-27 with the percentage of circulating Th17 cells and 
concentrations of plasma IL-17 in patients with progressive MS(Tang et al., 2015)

We found in this study that decreased IL-27 levels were associated with disease severity and total length of newly identified spinal cord lesion in patients with NMOSD. Together, these previous findings and the present work suggest that IL-27 plays a role in the clinical pathology of NMOSD. Specifically, we postulate that IL-27 may act via similar mechanisms in NMOSD as it does in MS to inhibit Th17 cell production and decrease pro-inflammatory cytokine levels.

Likewise, IL-35, a newly identified IL-12 family member, mainly suppresses inflammatory responses(Chaturvedi et al., 2013; Collion et al., 2007). However, studies indicating that IL-35 is an inhibitor of inflammation come mostly from work in mouse models or findings from healthy individuals. Thus, the role of IL-35 in autoimmune diseases remains to be defined. In this study, we observed dramatically decreased serum IL-35 levels in patients with NMOSD compared with matched healthy controls, suggesting that IL-35 may be involved in disease pathogenesis. It has been reported that Treg cells were reduced in NMOSD(Lehmann-Horn et al., 2011). Considering that IL-35 is an anti-inflammatory cytokine that is mainly produced by Treg cells, we speculate that decreased IL-35 levels may be attributable to the decline of Treg cells in patients with NMOSD in relapse phases. Of particular note, serum IL-35 concentrations were negatively correlated with EDSS, implying that IL-35 may be a biomarker that is reflective of NMOSD severity. IL-35 inhibits inflammation mainly by inhibiting proliferation of CD4+ effector T cells (Teff cells, including Th1, Th2, Th17), decreasing secretion of Th1-type cytokines, decreasing B cell proliferation, and inducing the production of inducible IL-35-producing Tregs (iTR35 cells) by suppressing the expression of the Treg transcription factor forkhead box P3 (Foxp3)(Collison et al., 2010; Collison et al., 2007; Wang et al., 2014). Thus, reduced IL-35 may lead to the development and exacerbation of inflammation inNMOSD via the downregulation of these pro-inflammatory processes.

Taken together, our data suggest that imbalances in the levels of IL-12 family member cytokines such as IL-27 and IL-35, may be involved in the pathogenesis of NMOSD. The IL-12 family participates in a variety of host immunity processes, 
suggesting that it may include important therapeutic targets for many autoimmune diseases. IL-35 is a particularly compelling target as recent studies have now shown that not only T-cells, but also B-cells (which are regulators of immune processes in autoimmune diseases), secrete IL-35(Shen et al., 2014; Wang et al., 2014). Mutant mice that do not express IL-35 in B-cells fail to recover from the demyelinating experimental autoimmune encephalitis (EAE), but are markedly resistant to Salmonella enterica serovar Typhimurium infection(Shen et al., 2014). In the experimental autoimmune uveitis model (EAU), IL-35 treatment prevents uveitis, while mice lacking IL-35 develop more severe uveitis(Wang et al., 2014). Notably, IL-35 can induce Breg proliferation in vivo and help Bregs convert into i35-Bregs. Moreover, IL-35 promotes the conversion of human B-cells into Bregs, indicating that this function of IL-35 is evolutionarily conserved between humans and mice(Shen et al., 2014).

\section{Conclusions}

In conclusion, our study showed that serum IL-27 and IL-35 levels were lower in patients with NMOSD. Clinically, serum IL-27 and IL-35 levels may be important biomarkers for the severity of NMOSD. IL-27 or IL-35 cytokines and other novel fusokines might represent a novel class of therapeutic cytokines for treating NMOSD.

\section{Acknowledgements}

This study was supported in part by the Tianjin Research Program of Application Foundation and Advanced Technology (15JCZDJC35700), the National Science Foundation of China (81471221, 81171183 to L.Y.) and the National Key Clinical Specialty Construction Project of China. 


\section{References}

Batten M, Li J, Yi S, Kljavin NM, Danilenko DM, Lucas S, et al. Interleukin 27 limits autoimmune encephalomyelitis by suppressing the development of interleukin 17-producing T cells. Nat Immunol. 2006;7:929-36.

Becher B, Durell BG, Noelle RJ. IL-23 produced by CNS-resident cells controls T cell encephalitogenicity during the effector phase of experimental autoimmune encephalomyelitis. J Clin Invest.

2003;112:1186-91.

Collison LW, Chaturvedi V, Henderson AL, Giacomin PR, Guy C, Bankoti J, et al. IL-35-mediated induction of a potent regulatory T cell population. Nat Immunol. 2010;11:1093-101.

Collison LW, Workman CJ, Kuo TT, Boyd K, Wang Y, Vignali KM, et al. The inhibitory cytokine IL-35 contributes to regulatory T-cell function. Nature. 2007;450:566-9.

Comabella M, Balashov K, Issazadeh S, Smith D, Weiner HL, Khoury SJ. Elevated interleukin-12 in progressive multiple sclerosis correlates with disease activity and is normalized by pulse cyclophosphamide therapy. J Clin Invest. 1998;102:671-8.

Cox JH, Kljavin NM, Ramamoorthi N, Diehl L, Batten M, Ghilardi N. IL-27 promotes T cell-dependent colitis through multiple mechanisms. J Exp Med. 2011;208:115-23.

Cua DJ, Sherlock J, Chen Y, Murphy CA, Joyce B, Seymour B, et al. Interleukin-23 rather than interleukin-12 is the critical cytokine for autoimmune inflammation of the brain. Nature. 2003;421:744-8.

Fitzgerald DC, Zhang GX, El-Behi M, Fonseca-Kelly Z, Li H, Yu S, et al. Suppression of autoimmune inflammation of the central nervous system by interleukin 10 secreted by interleukin 27 -stimulated $T$ cells. Nat Immunol. 2007;8:1372-9.

Jarius S, Wildemann B. AQP4 antibodies in neuromyelitis optica: diagnostic and pathogenetic relevance. Nat Rev Neurol. 2010;6:383-92.

Lehmann-Horn K, Schleich E, Hertzenberg D, Hapfelmeier A, Kumpfel T, von Bubnoff N, et al. Anti-CD20 B-cell depletion enhances monocyte reactivity in neuroimmunological disorders. J Neuroinflammation. 2011;8:146.

Lennon VA, Wingerchuk DM, Kryzer TJ, Pittock SJ, Lucchinetti CF, Fujihara K, et al. A serum autoantibody marker of neuromyelitis optica: distinction from multiple sclerosis. Lancet. 2004;364:2106-12.

Li TT, Zhang T, Chen GM, Zhu QQ, Tao JH, Pan HF, et al. Low level of serum interleukin 27 in patients with systemic lupus erythematosus. J Investig Med. 2010;58:737-9.

Matsushita T, Tateishi T, Isobe N, Yonekawa T, Yamasaki R, Matsuse D, et al. Characteristic cerebrospinal fluid cytokine/chemokine profiles in neuromyelitis optica, relapsing remitting or primary progressive multiple sclerosis. PLoS One. 2013;8:e61835.

Shen P, Roch T, Lampropoulou V, O'Connor RA, Stervbo U, Hilgenberg E, et al. IL-35-producing B cells are critical regulators of immunity during autoimmune and infectious diseases. Nature.

2014;507:366-70.

Stumhofer JS, Laurence A, Wilson EH, Huang E, Tato CM, Johnson LM, et al. Interleukin 27 negatively regulates the development of interleukin 17-producing $T$ helper cells during chronic inflammation of the central nervous system. Nat Immunol. 2006;7:937-45.

Sun L, He C, Nair L, Yeung J, Egwuagu CE. Interleukin 12 (IL-12) family cytokines: Role in immune pathogenesis and treatment of CNS autoimmune disease. Cytokine. 2015;75:249-55. 
Tang SC, Fan XH, Pan QM, Sun QS, Liu Y. Decreased expression of IL-27 and its correlation with Th1 and Th17 cells in progressive multiple sclerosis. J Neurol Sci. 2015;348:174-80.

Uzawa A, Mori M, Arai K, Sato Y, Hayakawa S, Masuda S, et al. Cytokine and chemokine profiles in neuromyelitis optica: significance of interleukin-6. Mult Scler. 2010;16:1443-52.

Uzawa A, Mori M, Kuwabara S. Cytokines and chemokines in neuromyelitis optica: pathogenetic and therapeutic implications. Brain Pathol. 2014;24:67-73.

Vignali DA, Kuchroo VK. IL-12 family cytokines: immunological playmakers. Nat Immunol.

2012;13:722-8.

Wang HH, Dai YQ, Qiu W, Lu ZQ, Peng FH, Wang YG, et al. Interleukin-17-secreting T cells in neuromyelitis optica and multiple sclerosis during relapse. J Clin Neurosci. 2011;18:1313-7. Wang J, Li X, Zhang DQ, Yang CS, Qi Y, Li MS, et al. Quantitative analysis of aquaporin-4 antibody in longitudinally extensive transverse myelitis. J Neuroimmunol. 2015;278:26-9.

Wang RX, Yu CR, Dambuza IM, Mahdi RM, Dolinska MB, Sergeev YV, et al. Interleukin-35 induces regulatory B cells that suppress autoimmune disease. Nat Med. 2014;20:633-41.

Wingerchuk DM, Banwell B, Bennett JL, Cabre P, Carroll W, Chitnis T, et al. International consensus diagnostic criteria for neuromyelitis optica spectrum disorders. Neurology. 2015;85:177-89.

Wong CK, Chen da P, Tam LS, Li EK, Yin YB, Lam CW. Effects of inflammatory cytokine IL-27 on the activation of fibroblast-like synoviocytes in rheumatoid arthritis. Arthritis Res Ther. 2010;12:R129. Yang CS, Zhang DQ, Wang JH, Jin WN, Li MS, Liu J, et al. Clinical features and sera anti-aquaporin 4 antibody positivity in patients with demyelinating disorders of the central nervous system from Tianjin, China. CNS Neurosci Ther. 2014;20:32-9.

Zhong X, Wang H, Dai Y, Wu A, Bao J, Xu W, et al. Cerebrospinal fluid levels of CXCL13 are elevated in neuromyelitis optica. J Neuroimmunol. 2011;240-241:104-8. 
Table 1. Characteristics of subjects with NMOSD

\section{Clinical characteristics}

Age at sampling (years)

$\operatorname{Sex}(F: M)$

Disease duration at the last follow-up (years)

Disease duration at sampling (years)

Annual relapse rate during the disease course (number of relapses per

year)

EDSS at sampling

Attack sites at sampling

No. of patients with optic neuritis

No. of patients with acute myelitis

No. of patients with intracranial lesions

MRI Total length of newly identified spinal cord lesion length at sampling (vertebral segments)

No. of patients with optic neuritis

No. of patients with MRI cerebral lesions

No. of patients with serum AQP4 antibody-positive at sampling (\%)

CSF (No.)

No. of cell (per $\mu \mathrm{L})$

Protein (mg/dl)

No. of patients with oligoclonal band-positive at sampling
NMOSD $(n=45)$

$46.71 \pm 13.24(20-74)$

$37: 8$

$8.80 \pm 6.83(2.00-36.00)$

$4.38 \pm 6.08(0-24.00)$

$0.58 \pm 0.33$

$4.90 \pm 2.01$

$4 / 45(8.89 \%)$

$45 / 45(100 \%)$

$11 / 45(24.44 \%)$

$6.60 \pm 4.09(2-18)$

$35 / 45(77.78 \%)$

31/43 (72.09\%)

$36 / 45(80.00 \%)$

$21 / 45$

$30.10 \pm 73.44(0-276)$

$45.90 \pm 22.06$

$1 / 21$ 


\section{Figure legends}

Fig. 1. Serum IL-27 levels in patients with NMOSD and healthy controls.

Fig. 2. Serum IL-35 levels in patients with NMOSD and healthy controls.

Fig. 3. The relationship between serum IL-27 levels and clinical parameters in NMOSD. (A) The correlation between serum IL-27 levels and EDSS in patients with NMOSD. (B) The correlation between serum IL-27 levels and EDSS in 12 patients with their first attack of NMOSD. (C) The correlation between serum IL-27 levels and spinal cord lesion length in patients with NMOSD. (D) The correlation between serum IL-27 levels and annual relapse rate in patients with NMOSD.

Fig. 4. The relationship between serum IL-35 levels and clinical parameters in NMOSD. (A) The relationship between serum IL-35 levels and EDSS in patients with NMOSD. (B) The correlation between serum IL-35 levels and EDSS in 12 patients with their first attack of NMOSD. (C) The relationship between serum IL-35 levels and annual relapse rate in patients with NMOSD. (D) The relationship between serum IL-35 levels and spinal cord lesion length in patients with NMOSD. 


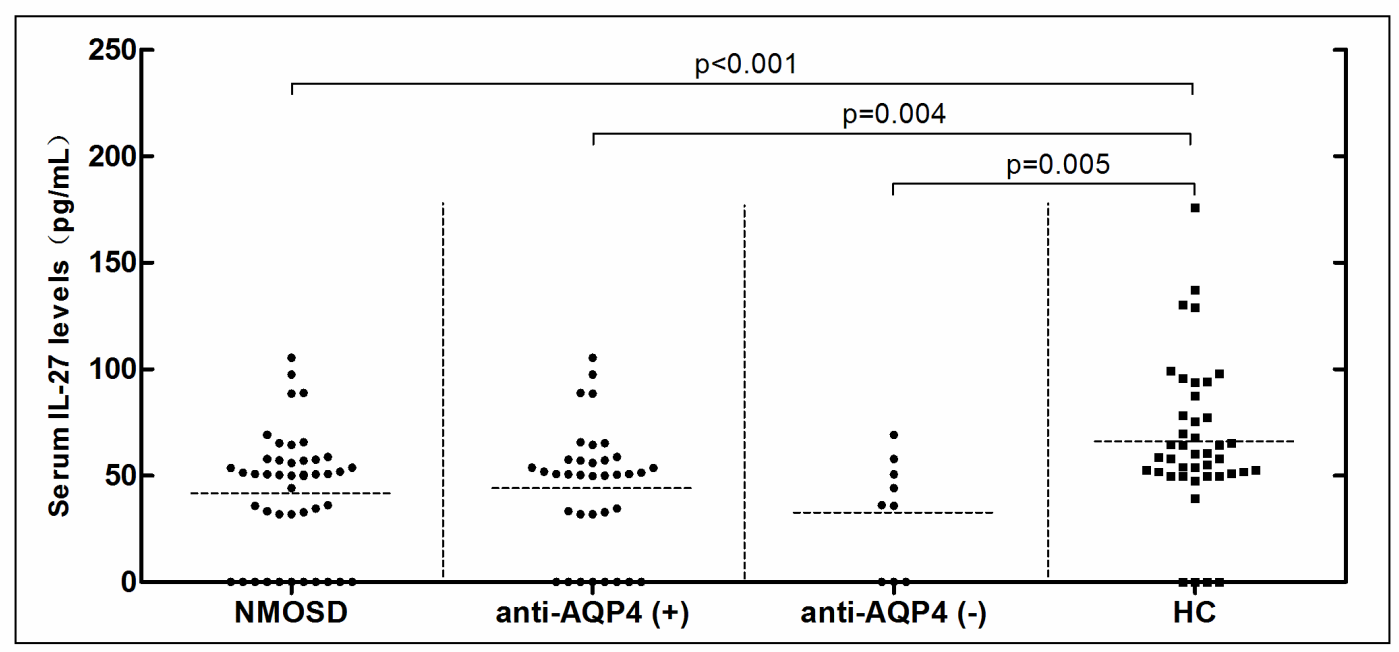




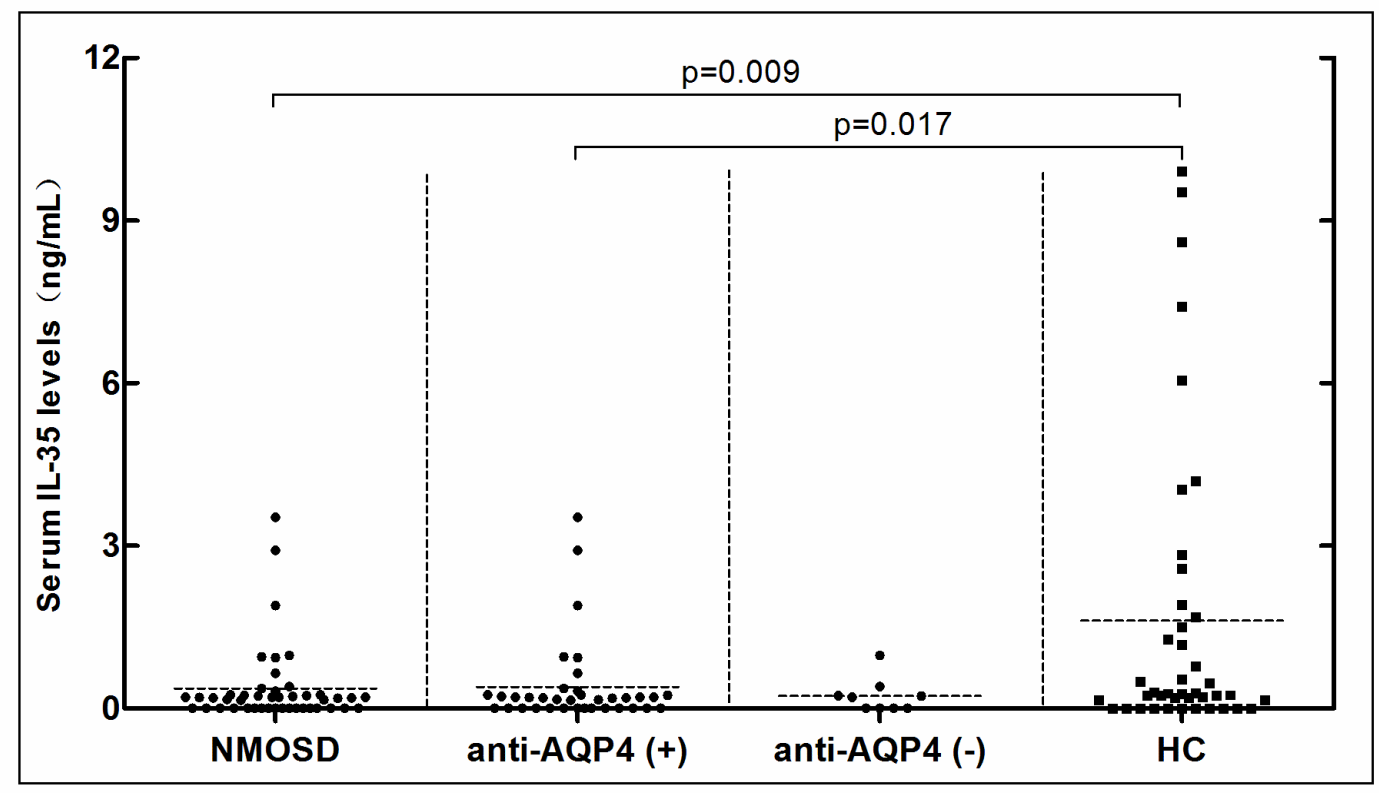




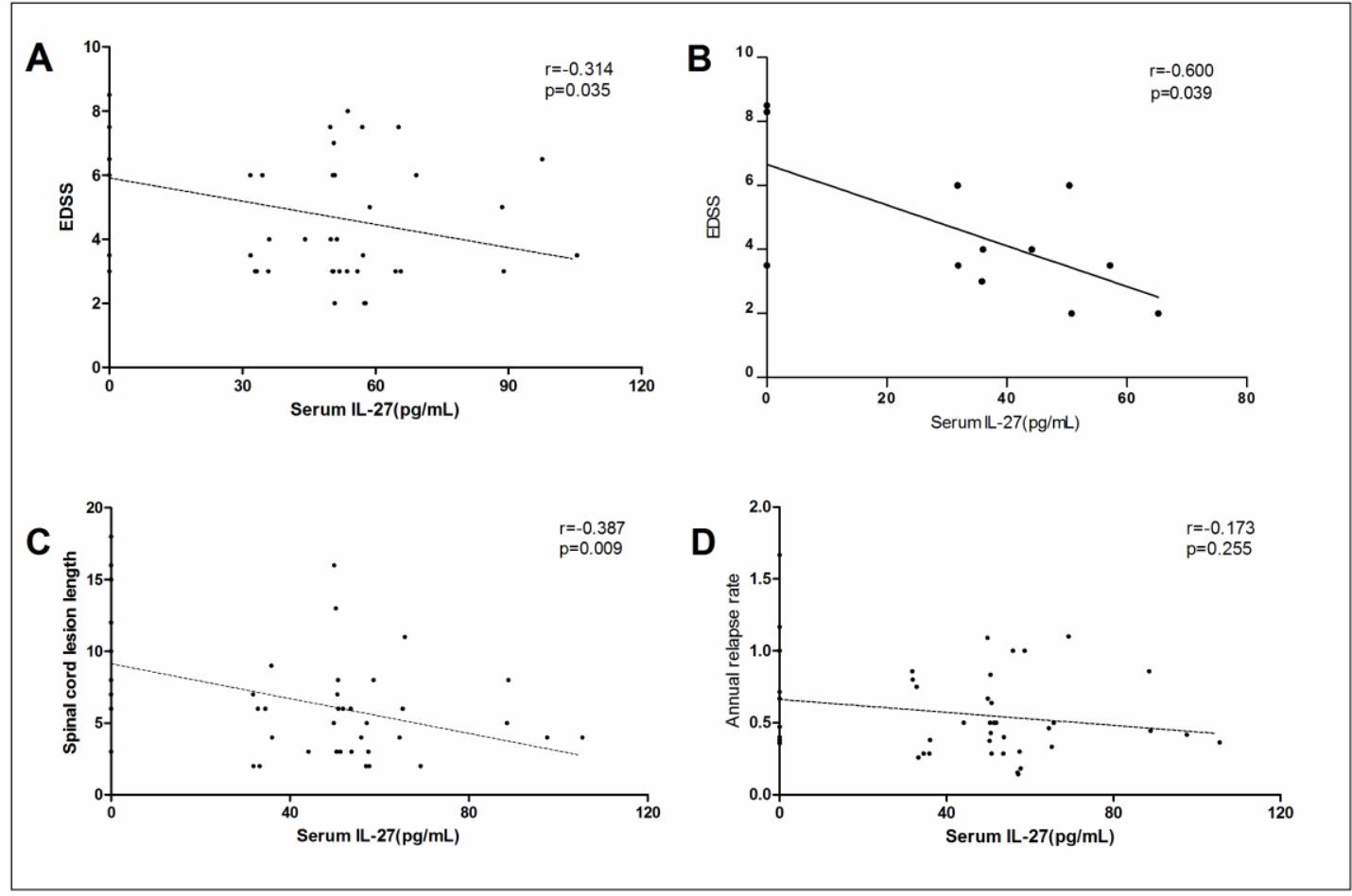




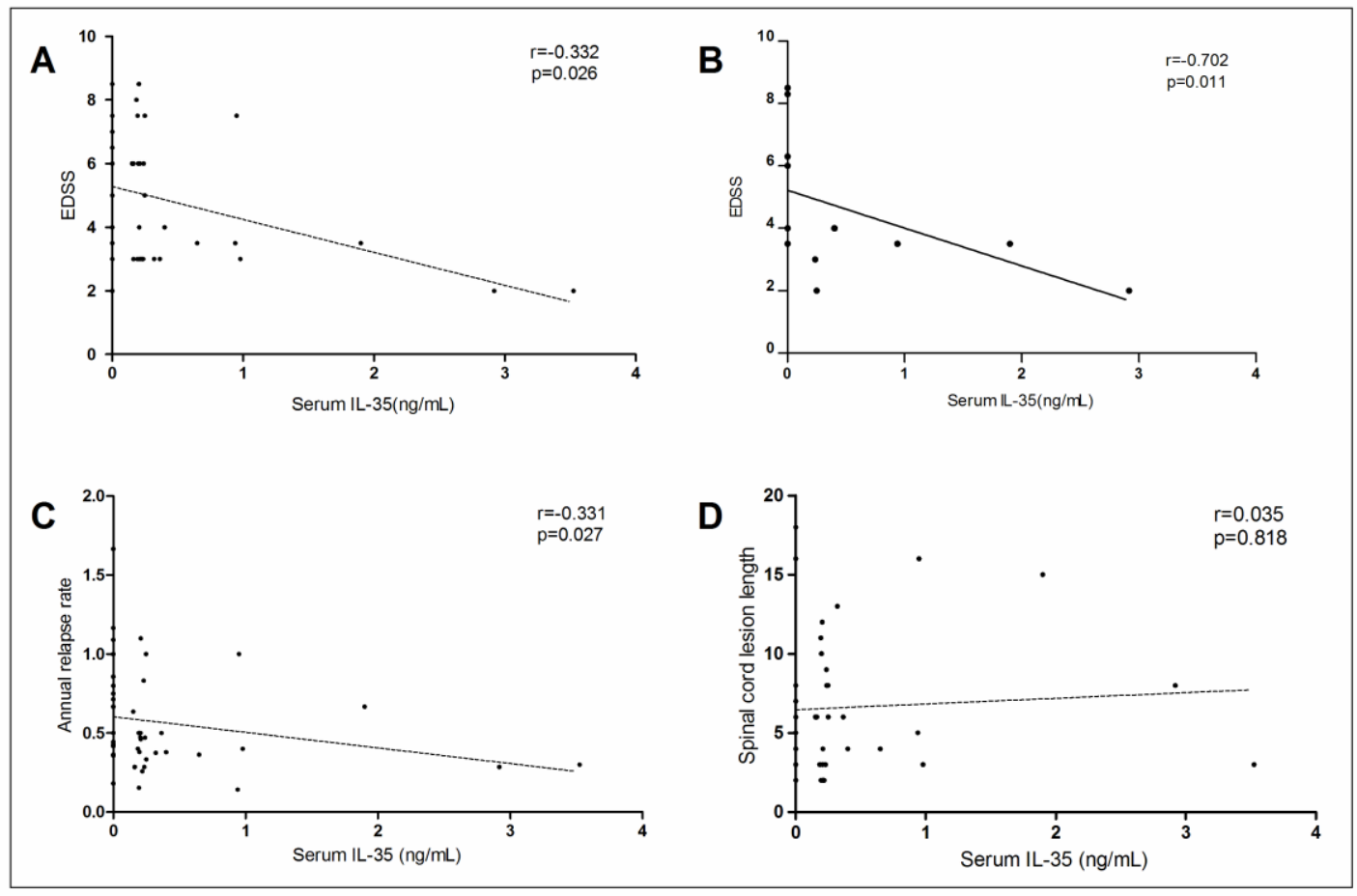




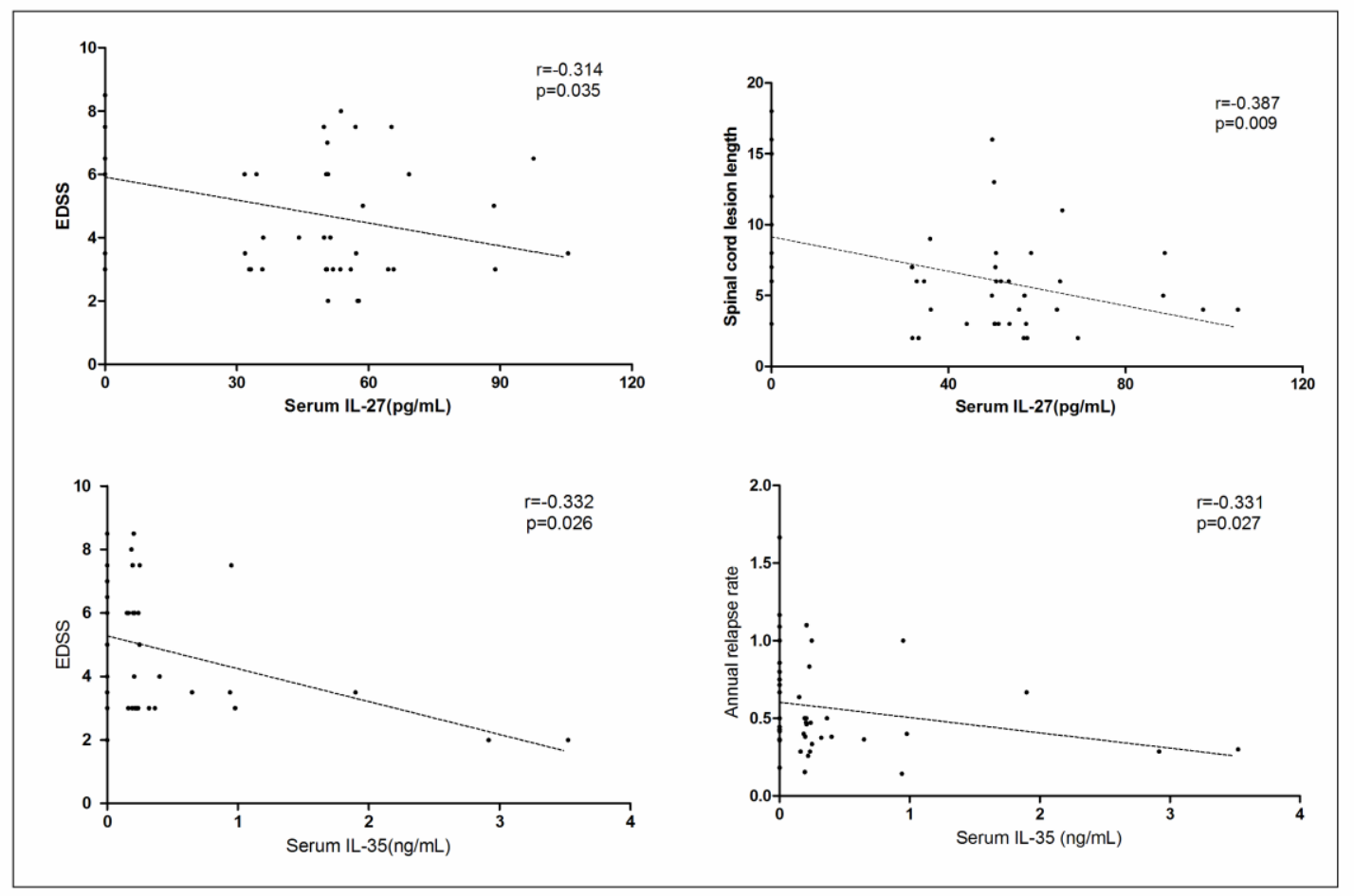

